\title{
Genes Associated with Thoracic Aortic Aneurysm and Dissection: 2019 Update and Clinical Implications
}

\author{
Thais Faggion Vinholo, MSc ${ }^{1}$ Adam J. Brownstein, $\mathrm{MD}^{2}$ Bulat A. Ziganshin, MD, $\mathrm{PhD}^{1,3}$ \\ Mohammad A. Zafar, MD ${ }^{1}$ Helena Kuivaniemi, MD, PhD ${ }^{4}$ Simon C. Body, MD, MPH ${ }^{5}$ \\ Allen E. Bale, MD $^{6}$ John A. Elefteriades, MD, PhD (hon) ${ }^{1}$
}

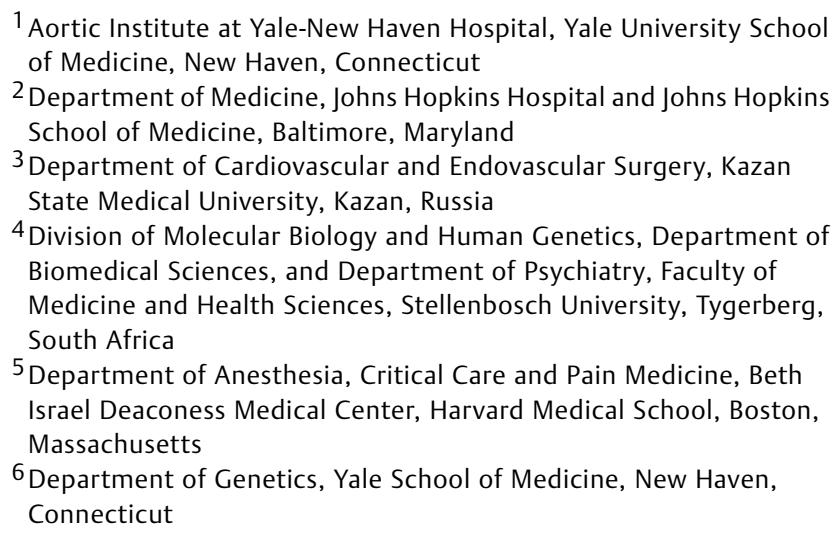

Address for correspondence John A. Elefteriades, MD, PhD (hon), Aortic Institute at Yale-New Haven, Yale University School of Medicine, 789 Howard Avenue, Clinic Building-CB317 New Haven, CT 06519 (e-mail: john.elefteriades@yale.edu).

\begin{abstract}
Keywords

- genetics

- thoracic aortic aneurysm

- aortic dissection

Thoracic aortic aneurysm is a typically silent disease characterized by a lethal natural history. Since the discovery of the familial nature of thoracic aortic aneurysm and dissection (TAAD) almost 2 decades ago, our understanding of the genetics of this disorder has undergone a transformative amplification. To date, at least 37 TAADcausing genes have been identified and an estimated $30 \%$ of the patients with familial nonsyndromic TAAD harbor a pathogenic mutation in one of these genes. In this review, we present our yearly update summarizing the genes associated with TAAD and the ensuing clinical implications for surgical intervention. Molecular genetics will continue to bolster this burgeoning catalog of culprit genes, enabling the provision of personalized aortic care.
\end{abstract}

\section{Introduction}

This review presents an annual update to the article "Genes Associated with Thoracic Aortic Aneurysm and Dissection: Update and Clinical Implications" originally published in 2017 and updated in 2018 in AORTA., ${ }^{1,2}$ We have updated the list of genes with identified genetic variants predisposing individuals to a thoracic aortic aneurysm or dissection (TAAD) in - Table 1, and the recommendation for individualized surgical interventions for specific genetic mutations is presented in - Fig. 1.
Thoracic aortic aneurysm (TAA) affects $1 \%$ of the general population $^{3}$ and its natural history is to enlarge an average of $0.14 \mathrm{~cm}$ per year. ${ }^{4}$ Prior to often lethal dissection or rupture, TAAs are usually asymptomatic. However, if identified and treated with appropriate blood pressure control and surgical intervention, life expectancy is improved.

Report of inherited TAAD in the $1990 \mathrm{~s}^{5}$ has led to the discovery and understanding of genetic and molecular mechanisms of TAAD. ${ }^{6}$ To date, variants in 37 genes have been associated with TAAD ( - Table 1; - Fig. 1). These genes explain approximately $30 \%$ of the familial nonsyndromic

Copyright $\odot 2019$ by Thieme Medical Publishers, Inc., 333 Seventh Avenue, New York, NY 10001, USA. Tel: +1(212) 584-4662.
License terms

cc) (i)
DOI https://doi.org/ 10.1055/s-0039-3400233. ISSN 2325-4637. 


\begin{tabular}{|c|c|c|c|c|c|c|c|c|c|c|c|c|c|c|c|c|c|c|c|c|}
\hline $\mid \sum$ & 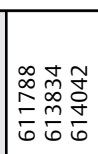 & 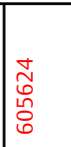 & 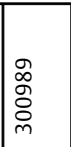 & 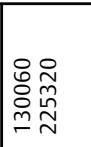 & 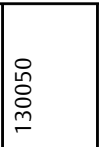 & 浐 & $\begin{array}{l}0 \\
\vdots \\
0 \\
m\end{array}$ & $\frac{\sqrt{\gamma}}{\frac{\gamma}{6}}$ & 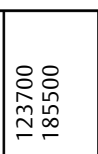 & 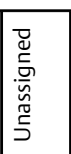 & \begin{tabular}{|l}
8 \\
$\vdots$ \\
$\vdots$ \\
\end{tabular} & 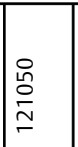 & 总 & $\left|\begin{array}{l}0 \\
0 \\
\frac{1}{6} \\
6\end{array}\right|$ & $\begin{array}{l}0 \\
0 \\
0 \\
0 \\
0\end{array}$ & $\begin{array}{l}\infty \\
\frac{0}{5} \\
\frac{1}{6}\end{array}$ & 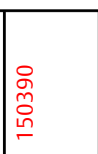 & $\begin{array}{l}0 \\
0 \\
0 \\
0 \\
0\end{array}$ & 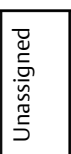 & $\mid \begin{array}{l}0 \\
\frac{6}{6} \\
\frac{6}{6}\end{array}$ \\
\hline 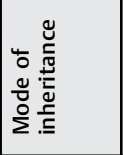 & \& & 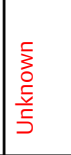 & 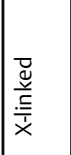 & $\begin{array}{l}\frac{⿱}{<} \\
+ \\
\stackrel{\alpha}{\alpha}\end{array}$ & \& & \& & \& & $\stackrel{\propto}{<}$ & \& & \& & \& & \& & $\overrightarrow{\vec{x}}$ & \& & \& & \& & \& & $\frac{\alpha}{\alpha}$ & \& & \& \\
\hline 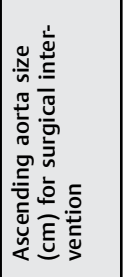 & 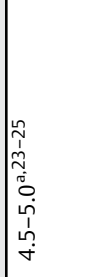 & 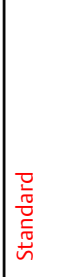 & 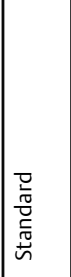 & 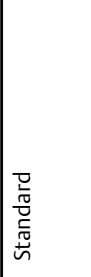 & 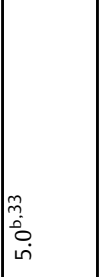 & 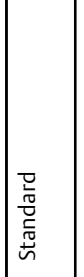 & 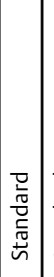 & 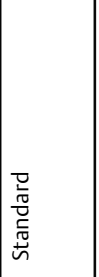 & 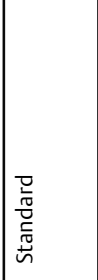 & 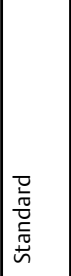 & 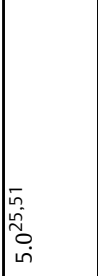 & 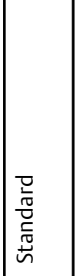 & 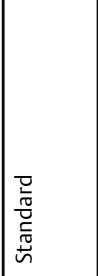 & 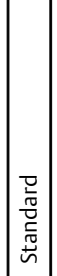 & 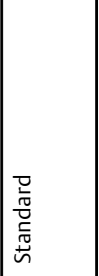 & 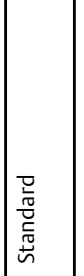 & 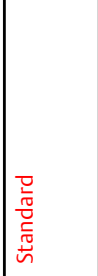 & 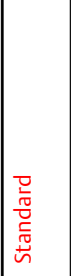 & 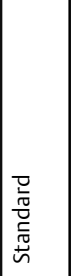 & 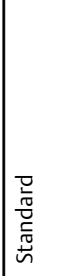 \\
\hline 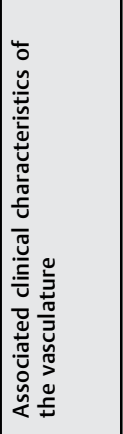 & 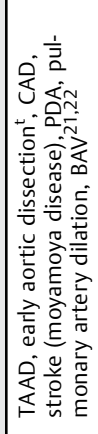 & 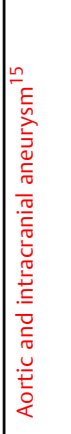 & 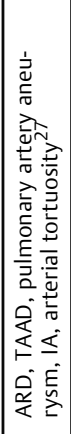 & 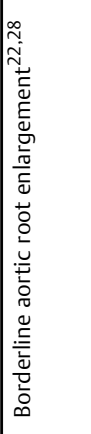 & 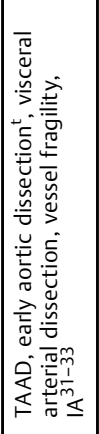 & 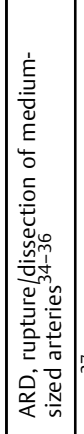 & . & 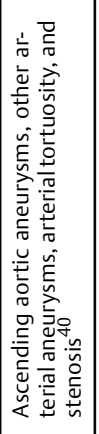 & 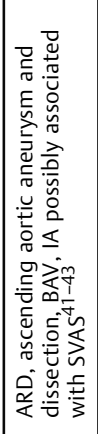 & 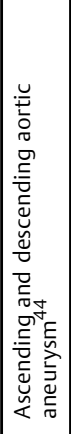 & 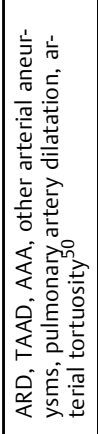 & 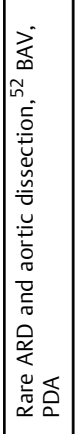 & 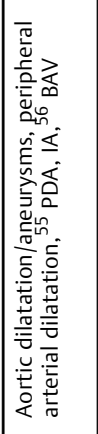 & 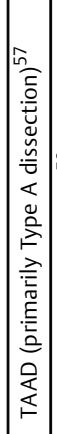 & 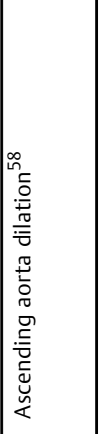 & 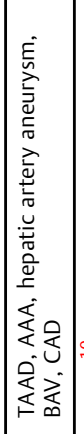 & $\mid$ & 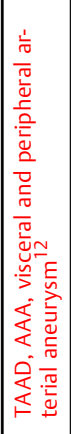 & 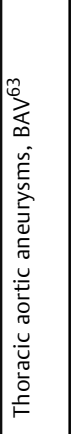 & 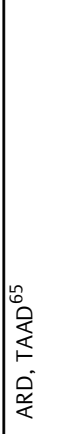 \\
\hline 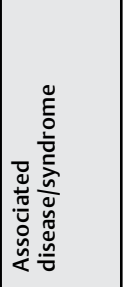 & 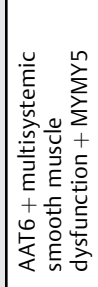 & 这 & 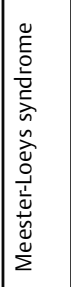 & 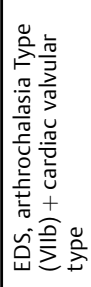 & 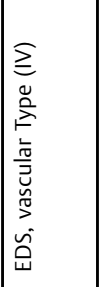 & 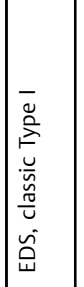 & 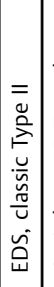 & 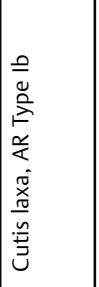 & 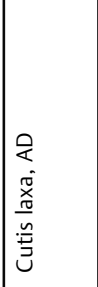 & 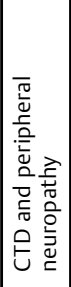 & 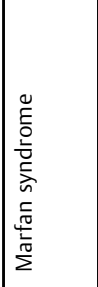 & 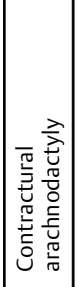 & 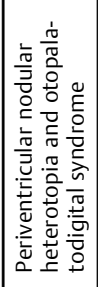 & E & 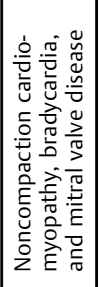 & \begin{tabular}{|l} 
\\
\\
$\frac{1}{4}$
\end{tabular} & 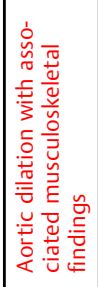 & 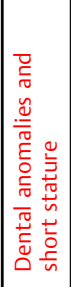 & 这 & 妾 \\
\hline 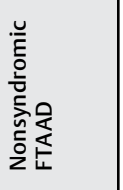 & + & + & 1 & 1 & 1 & 1 & 1 & 1 & 1 & 1 & + & 1 & 1 & + & + & + & 1 & 1 & + & + \\
\hline 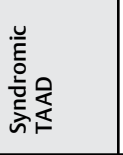 & + & + & + & + & + & + & + & + & + & + & + & + & + & 1 & 1 & 1 & + & + & 1 & 1 \\
\hline 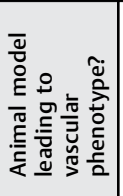 & 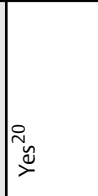 & $\stackrel{2}{2}$ & 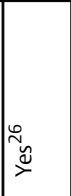 & in & 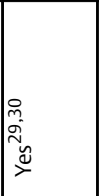 & $\frac{1}{2}$ & 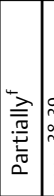 & 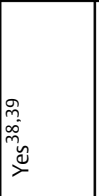 & $\stackrel{\circ}{2}$ & 2 & 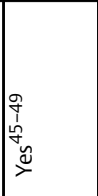 & 2 & {$\left[\begin{array}{l}n \\
y \\
y\end{array}\right.$} & 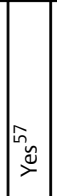 & 2 & 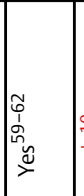 & 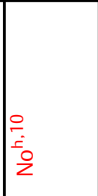 & 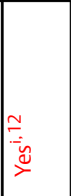 & 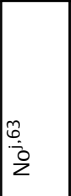 & 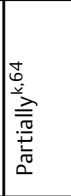 \\
\hline $\begin{array}{l}\text { 동 } \\
\stackrel{0}{0} \\
\stackrel{0}{2}\end{array}$ & 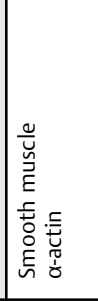 & 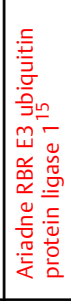 & 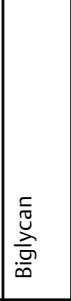 & 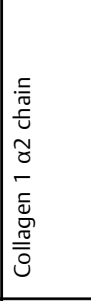 & 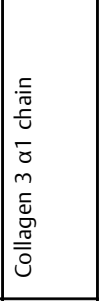 & 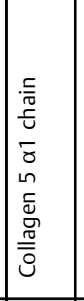 & 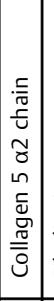 & 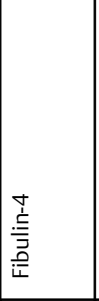 & \begin{tabular}{|l} 
\\
$\frac{5}{5}$ \\
$\frac{D}{W}$ \\
\end{tabular} & 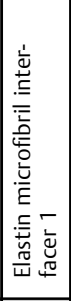 & 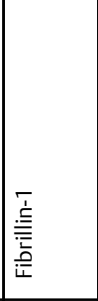 & 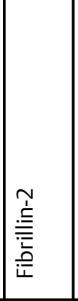 & 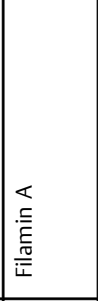 & 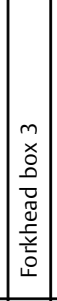 & 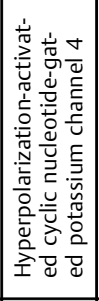 & 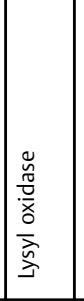 & 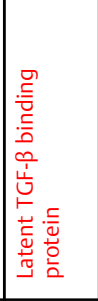 & 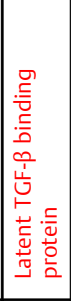 & 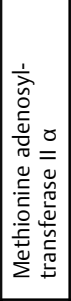 & 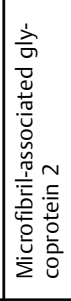 \\
\hline 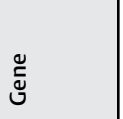 & 莡 & $\mid$\begin{tabular}{|c}
$\frac{7}{2}$ \\
妾
\end{tabular} & 孞 & 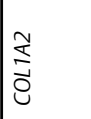 & 齐 & 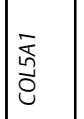 & 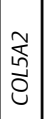 & $\frac{\varrho}{\Sigma}$ & z & 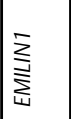 & $\sum_{\substack{0\\
}}$ & 部 & $\sum_{i=1}^{\infty}$ & $\mid \begin{array}{l}\hat{y} \\
\hat{z} \\
0\end{array}$ & $\mid \begin{array}{l}\dot{z} \\
\text { In }\end{array}$ & ঐِ & 产 & 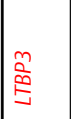 & $\mid \frac{\sqrt{3}}{\frac{d}{d}}$ & $\mid \frac{\hat{n}}{\hat{\alpha}}$ \\
\hline
\end{tabular}




\begin{tabular}{|c|c|c|c|c|c|c|c|c|c|c|c|c|c|c|c|c|c|c|c|}
\hline $\mid \sum_{0}^{\Sigma}$ & $\begin{array}{l}\stackrel{8}{0} \\
\stackrel{2}{0} \\
\end{array}$ & $\mid$ & $\begin{array}{c}0 \\
0 \\
0 \\
0 \\
0\end{array}$ & $\mid$ & 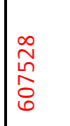 & 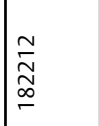 & 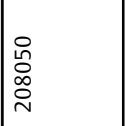 & 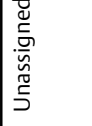 & 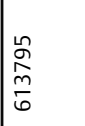 & 总 & $\begin{array}{l}\overline{\tilde{O}} \\
\tilde{\tilde{O}} \\
0\end{array}$ & $\mid \begin{array}{l}\infty \\
\infty \\
\infty \\
\infty \\
-\infty\end{array}$ & $\begin{array}{l}\stackrel{0}{0} \\
\text { 岕 } \\
\text { m }\end{array}$ & $\mid$ & م & & $\frac{\sigma}{\sigma}$ & $\mid \begin{array}{l}\infty \\
0 \\
0 \\
0 \\
0\end{array}$ & 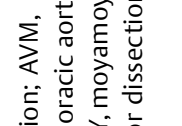 \\
\hline 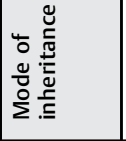 & \& & \& & \& & \& & 安 & \& & $\stackrel{\sim}{<}$ & \& & \& & षे & \& & $\vec{x}$ & $\vec{x}$ & \& & \& & & \& & \& & 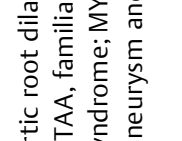 \\
\hline 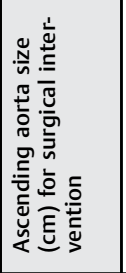 & 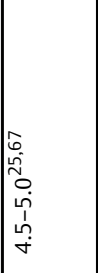 & 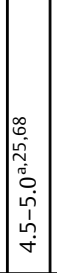 & 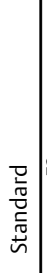 & 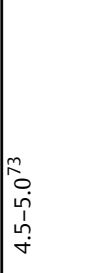 & 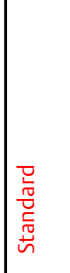 & 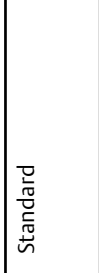 & 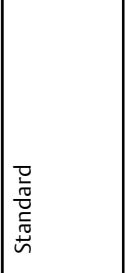 & 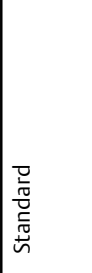 & $\mid$ & 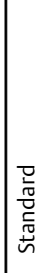 & 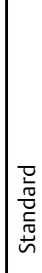 & 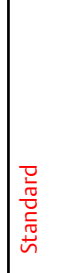 & 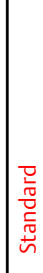 & 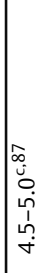 & $\frac{\sqrt{n}}{d}$ & & 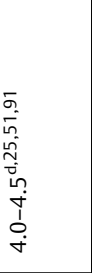 & 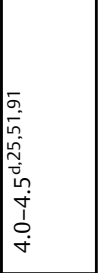 & 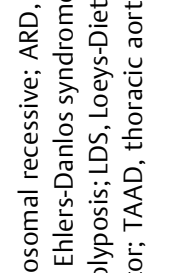 \\
\hline 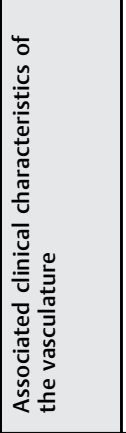 & 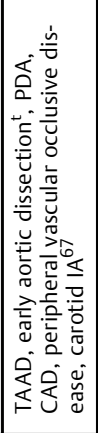 & 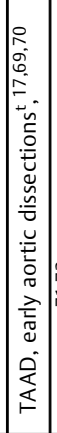 & 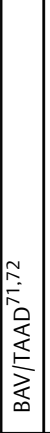 & 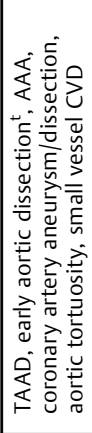 & $\mid$ & 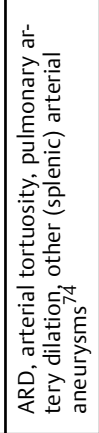 & 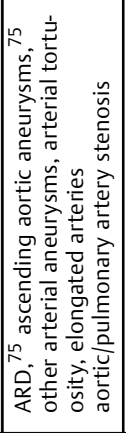 & 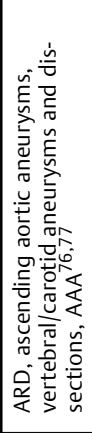 & 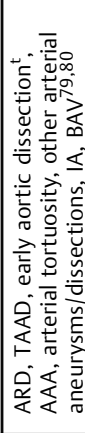 & 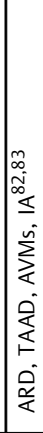 & 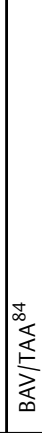 & 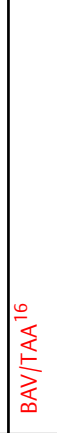 & $\mid \begin{array}{c}\frac{0}{2} \\
\frac{5}{5} \\
\frac{5}{2} \\
\infty\end{array}$ & 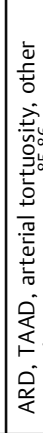 & & & 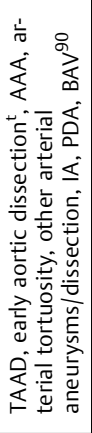 & 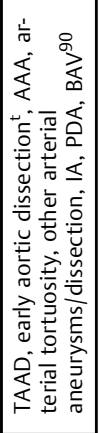 & 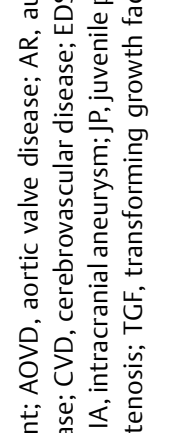 \\
\hline 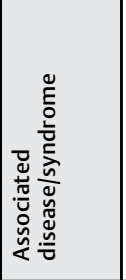 & $\frac{8}{8}$ & 妾 & $\begin{array}{l}\overline{0} \\
0 \\
0 \\
\end{array}$ & $\mid \frac{\infty}{4}$ & $\vec{\alpha}$ & 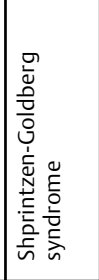 & 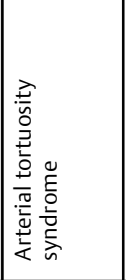 & 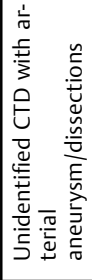 & 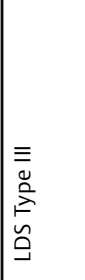 & 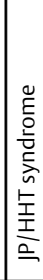 & 余 & 召 & 产 & 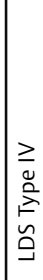 & ב & & 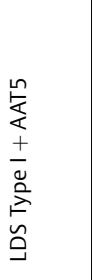 & 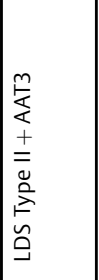 & 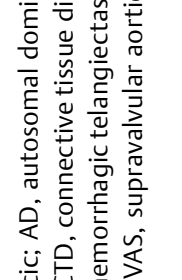 \\
\hline 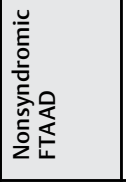 & + & + & + & + & + & 1 & 1 & 1 & + & 1 & + & 1 & & + & & & + & + & 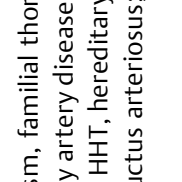 \\
\hline 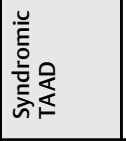 & 1 & 1 & 1 & 1 & 1 & $1+$ & + & + & + & + & 1 & + & & + & + & & + & + & 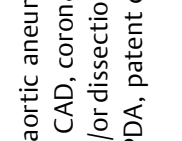 \\
\hline 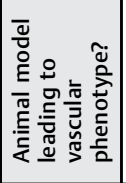 & 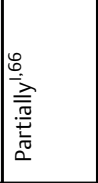 & 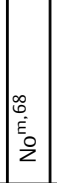 & 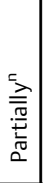 & z & $\stackrel{\check{\nu}}{\check{\nu}}$ & $i_{2}^{\circ}$ & $\frac{1}{2}$ & z & 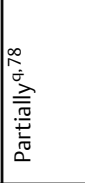 & 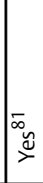 & $\stackrel{\circ}{2}$ & 2 & z & $\stackrel{\Perp}{\rightleftharpoons}$ & $z$ & & $\stackrel{u}{\check{u}}$ & . & 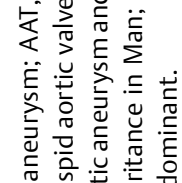 \\
\hline 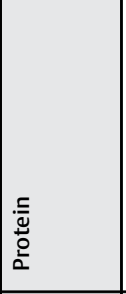 & 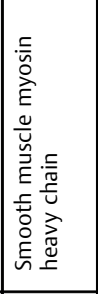 & 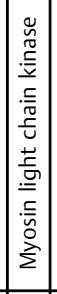 & \begin{tabular}{|l|}
\multicolumn{1}{|c}{} \\
\\
$\mathbf{0}$ \\
2 \\
\end{tabular} & 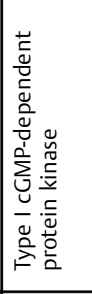 & 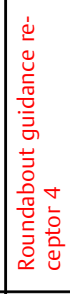 & 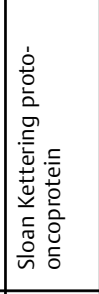 & 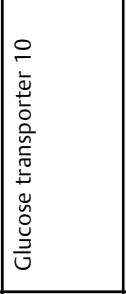 & 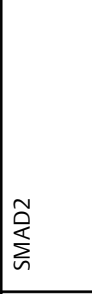 & 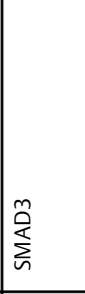 & 草 & 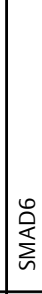 & 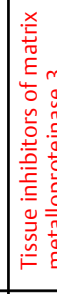 & 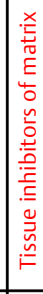 & 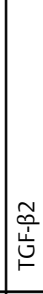 & & & 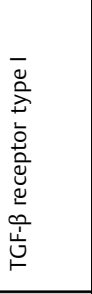 & 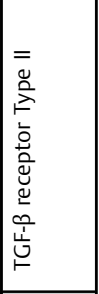 & 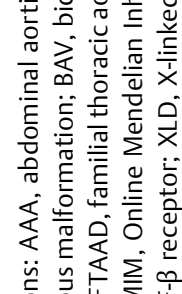 \\
\hline 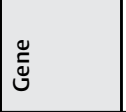 & 搽 & 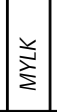 & \begin{tabular}{|l|}
$\begin{array}{l}1 \\
0 \\
0 \\
2\end{array}$ \\
\end{tabular} & 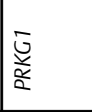 & $\mid$ & 㐫 & 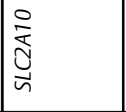 & 咅 & 竞 & in & 章 & 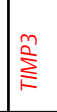 & $s$ & 5 & & & 畄 & 琶 & 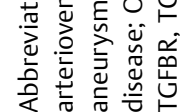 \\
\hline
\end{tabular}




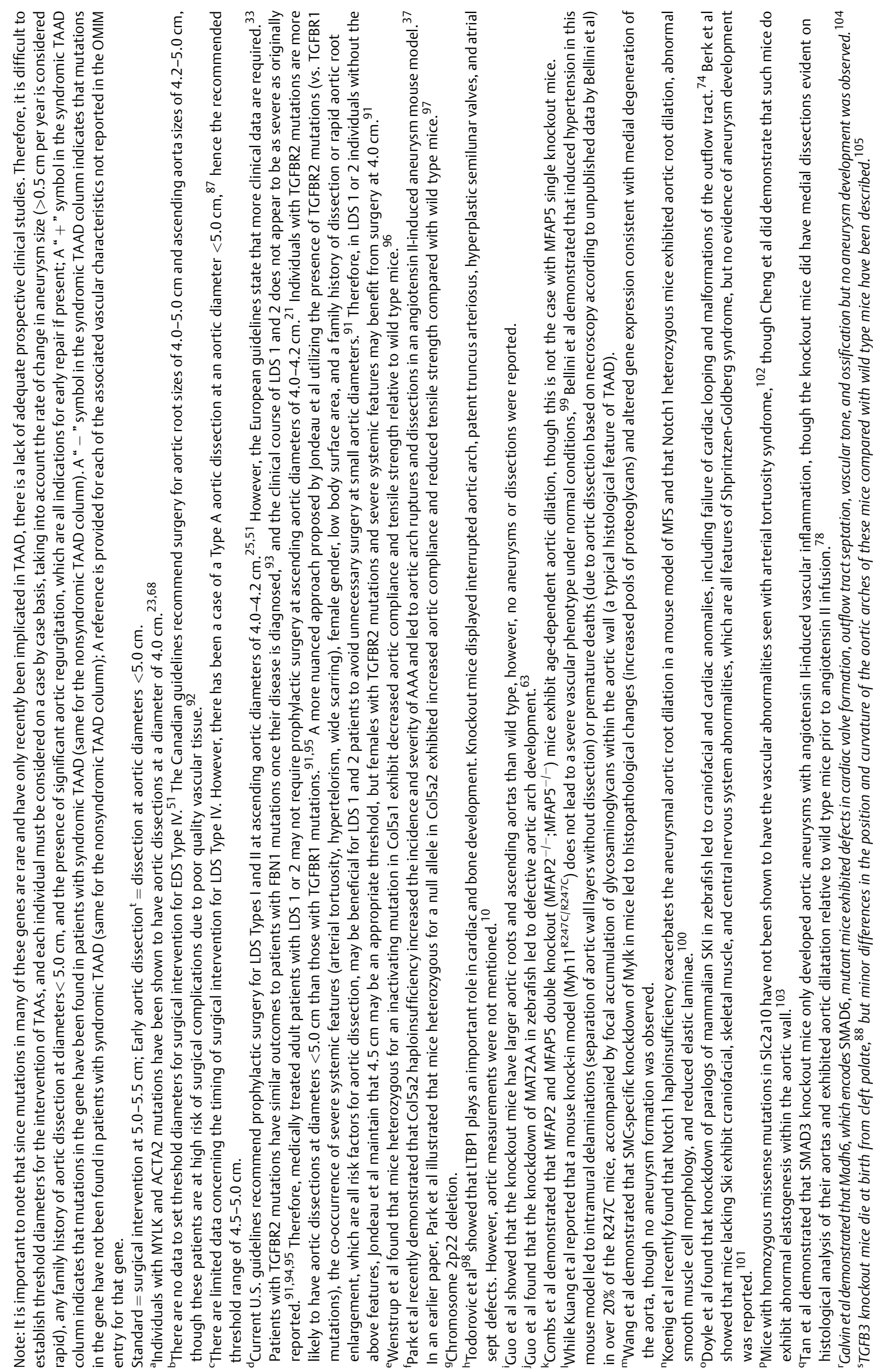




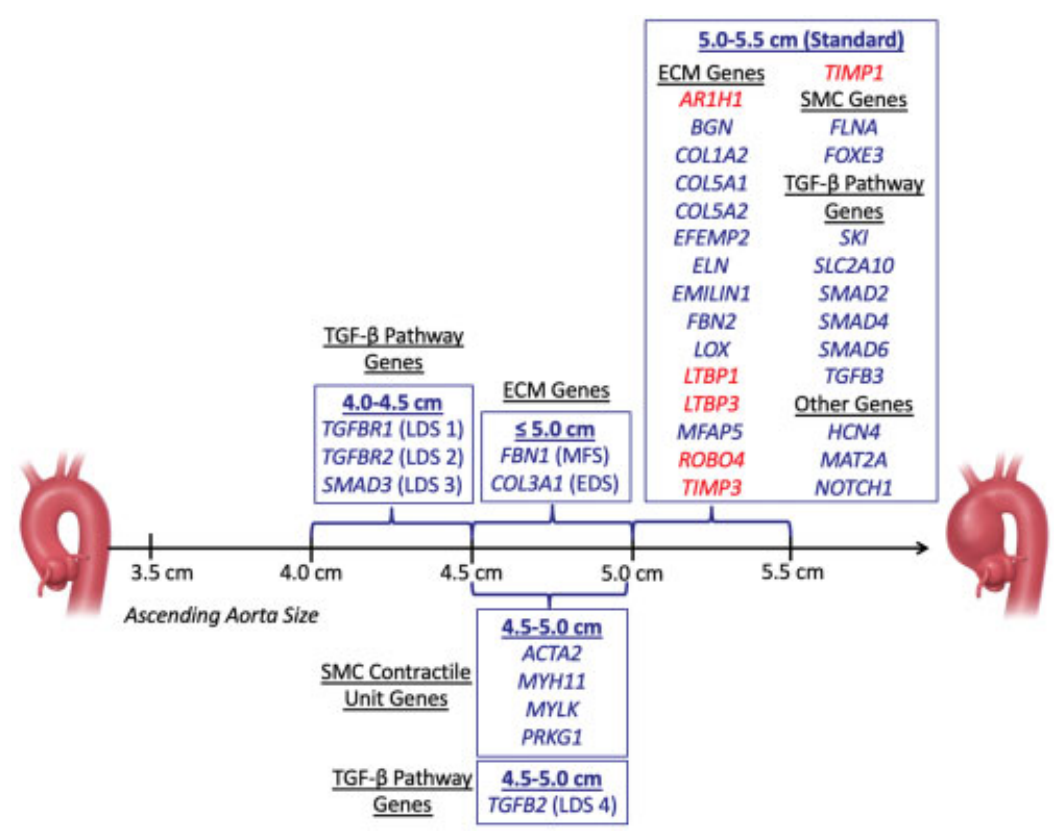

Fig. 1 Ascending aortic dimensions for prophylactic surgical intervention (Data derived from - Table 1 and modified with permission from Brownstein et $\mathrm{al}^{2}$ ). Any gene newly reported during the past year to be associated with TAAD is highlighted in red. ECM, extracellular matrix; SMC, smooth muscle cell; TGF, transforming growth factor.

TAAD. ${ }^{7}$ These genes encode proteins of the extracellular matrix, vascular smooth muscle cell contractile unit, or transforming growth factor $\beta$ (TGF- $\beta$ )-signaling pathways ${ }^{8}$ and thus are essential to the structure and maintenance of the aortic wall.

During 2018, several important studies were published that have enhanced our understanding of the pathogenesis of TAAD. Gould et $\mathrm{al}^{9}$ performed whole-exome sequencing (WES) and targeted sequencing on 736 individuals with bicuspid aortic valve (BAV), non-syndromic ascending aortic aneurysm (AscAA), and 376 controls. ${ }^{9}$ In $13(1.8 \%)$ of the affected individuals a heterozygous ROBO4 mutation was identified, including two variants that segregated with disease among two affected families. ${ }^{9} \mathrm{ROBO} 4$ is well expressed in vascular endothelial cells and plays a role in endothelial barrier function. ${ }^{9}$ In this study, its expression was found to be diminished in the resected aorta sample of an affected individual with AscAA. ${ }^{9}$ To further test their hypothesis that $\mathrm{ROBO4}$ variants lead to the disruption of endothelial performance at a cellular level, thus altering vascular permeability, the authors cultured human aortic endothelial cells and either silenced $\mathrm{ROBO}$ or expressed ROBO4 variants. They confirmed that ROBO4 abnormalities did indeed induce endothelial barrier dysfunction. Lastly, the authors created homozygous ROBO4 knockout mice and a knock-in mouse with an $\mathrm{ROBO4}$ splice donor site mutation; the affected mice presented with a mix of aortic valve dysfunction (BAV and/or aortic regurgitation or stenosis) and AscAA, confirming their suspicion that a heterozygous mutation in $\mathrm{ROBO} 4$ can lead to a nonsyndromic presentation of BAV/AscAA. ${ }^{9}$

Latent transforming growth factor binding proteins (LTBP), a family of extracellular matrix glycoproteins, have been shown to play a significant role in TGF- $\beta$ regulation. ${ }^{10}$
LTBP1, in particular, can bind to fibrillin-1 and inactivate TGF- $\beta .^{10,11}$ Quiñones-Pérez et al described a case series involving a three-generation family with TAA found to have a chromosome 2p22.3-p22.2 deletion involving LTBP1, amongst other genes. ${ }^{10}$ Despite multiple genes being involved in the deletion, LTBP1 was considered the likely culprit given its relationship to TGF- $\beta$. In addition to TAA, the affected individuals displayed additional features of Marfan syndrome (MFS) and Loeys-Dietz syndrome, even though none of them met the criteria for diagnosis.

Mutations of the latent TGF- $\beta$ binding protein-3 (LTBP3) gene have been associated with TAAD in a WES study of 271 individuals from unrelated families with heritable thoracic aortic disease (multiple affected family members) without a known genetic etiology for aortopathy. ${ }^{12}$ In this study, compound heterozygous variants in one family and a homozygous insertion/deletion variant in $L T B P 3$ in a second family were identified. Sequencing of 338 additional individuals with non-syndromic TAAD found nine additional heterozygous $L T B P 3$ rare variants. The authors also demonstrated that LTBP3 knockout mice manifested enlarged aortic roots and ascending aortas compared with wild type mice. These findings demonstrate that individuals with LTPB3 are at increased risk for TAAD, in addition to the already established risk for skeletal and dental abnormalities. ${ }^{12-14}$

Rare mutations in the Parkin-like E3 ubiquitin ligase Ariadne-1 (ARIH1) have been observed in patients with early-onset or familial TAAD. ${ }^{15}$ AR1H1 encodes a protein of the LINC (linker of nucleoskeleton and cytoskeleton), a protein complex essential for anchoring myocyte nuclei to the cytoskeleton. ${ }^{15}$ Aortic tissues from patients with these mutations exhibit affected nuclear morphology in vascular smooth muscle cells. 
It is well known there is an increased risk for BAV and TAA among individuals with Turner syndrome, although the precise etiology has thus far remained elusive. Corbitt et $\mathrm{al}^{16}$ demonstrated that Turner syndrome patients with putatively-deleterious mutations in TIMP3 are associated with a greater incidence of BAV and TAA than the patients without TIMP3 variants. Hemizygosity for coincident TIMP1/ TIMP3 variants, synergistically increased the risk for BAV and TAA, ${ }^{16}$ due to TIMP1's functional redundancy with TIMP3.

Numerous mutations of the myosin light chain kinase (MYLK) gene have been associated with TAAD. Shalata et al have identified an additional MYLK missense mutation in a single pedigree. ${ }^{17}$ Myosin light chain kinase phosphorylates myosin regulatory light chains to facilitate actin-myosin generation of contraction. The mutation was shown to be functional, reducing kinase activity.

Insights to the pathogenesis of TAAD are as important as identifying TAAD variants. Nogi et $\mathrm{al}^{18}$ found the protein expression of small GTP-binding protein GDP dissociation stimulator (SmgGDS) in aortic smooth muscle cells was decreased in TAAD patients compared with controls. ${ }^{18} \mathrm{SmgGDS}$ is encoded by the RAP1GDS1 gene and known to be involved in the contraction of vascular smooth muscle cell (VSMC). ${ }^{18}$ Using a heterozygous SmgGDS ${ }^{+-}$mouse model, since the complete knockout (SmgGDS ${ }^{-1-}$ ) was embryologically lethal, they observed that the downregulation of SmgGDS was causing "pathological phenotype changes in VSMC" via the angiotensin-II pathway. ${ }^{18}$ Furthermore, they demonstrated that when SmgGDS was overexpressed in the SmgGDS ${ }^{+/}$, the mice had less aortic growth and fewer aortic ruptures, suggesting that SmgGDS could be used as a biomarker or a therapeutic agent.

\section{Conclusion}

Advances in 2018 have increased our understanding of the pathogenesis of TAAD. The number of genes with genetic variants or mutations associated with TAAD has increased from 29 in our original 2017 report $^{2}$ to 37 in this 2019 update. Advances in genetic techniques and bioinformatics tools have enabled rapid progress in the genetic and molecular understanding of TAA. As the cost for genome sequencing decreases, we anticipate accelerating progress. With our greater understanding of the genetics of the individuals affected with TAAD and their specific genetic mutations or susceptibility variants, we can provide a personalized aortic care, tailoring surgical recommendations for each patient depending on their individual genetic profiles. Because most families that have multiple affected members with TAAD still have not had known genetic variants identified in the aortopathy genes, we expect many new genes harboring variants for TAAD will be discovered in the foreseeable future and thereby enhance our genetic dictionary. Furthermore, it is important to remind ourselves that every disease-causing mutation starts out as a variant of unknown significance (VUS). ${ }^{19}$ Only after extensive functional studies it is possible to confidently state that a VUS is a disease-causing mutation. Such work requires multidisciplinary collaboration.

We will continue to report annual updates regarding the "TAA genetic dictionary" with updates to the Table and
Figure below and provide suggested surgical intervention criteria for each identified mutation.

Funding

None.

Conflict of Interest

The authors declare no conflict of interest related to this article.

Acknowledgment

None.

\section{References}

1 Brownstein AJ, Kostiuk V, Ziganshin BA, et al. Genes associated with thoracic aortic aneurysm and dissection: 2018 update and clinical implications. Aorta (Stamford) 2018;6(01):13-20

2 Brownstein AJ, Ziganshin BA, Kuivaniemi H, Body SC, Bale AE, Elefteriades JA. Genes associated with thoracic aortic aneurysm and dissection: an update and clinical implications. Aorta (Stamford) $2017 ; 5(01): 11-20$

3 Verstraeten A, Luyckx I, Loeys B. Aetiology and management of hereditary aortopathy. Nat Rev Cardiol 2017;14(04):197-208

4 Zafar MA, Li Y, Rizzo JA, et al. Height alone, rather than body surface area, suffices for risk estimation in ascending aortic aneurysm. J Thorac Cardiovasc Surg 2018;155(05):1938-1950

5 Ziganshin BA, Elefteriades JA. Genetic impact on thoracic aortic aneurysms. In: Eskandari MK, Pearce WK, Yao JST, eds.Current Vascular Surgery: Northwestern Vascular Symposium. Raleigh, NC: PMPH USA; 2017:461480

6 Pinard A, Jones GT, Milewicz DM. Genetics of thoracic and abdominal aortic diseases. Circ Res 2019;124(04):588-606

7 Milewicz DM, Regalado E. Heritable thoracic aortic disease overview. In: Adam MP, Ardinger HH, Pagon RA, et al., eds. GeneReviews®. Seattle, WA1993

8 Elefteriades J, Brownstein AJ, Ziganshin BA. Clinical and molecular genetics of thoracic aortic aneurysm and dissection. In: Melissano G, Chiesa R, eds. Aortic Dissection: Patients True Stories and the Innovations that Saved Their Lives. Milan, Italy: Edi.Ermes; 2016:49-79

9 Gould RA, Aziz H, Woods CE, et al; Baylor-Hopkins Center for Mendelian Genomics; MIBAVA Leducq Consortium. ROBO4 variants predispose individuals to bicuspid aortic valve and thoracic aortic aneurysm. Nat Genet 2019;51(01):42-50

10 Quiñones-Pérez B, VanNoy GE, Towne MC, et al. Three-generation family with novel contiguous gene deletion on chromosome 2 p22 associated with thoracic aortic aneurysm syndrome. Am J Med Genet A 2018;176(03):560-569

11 Takeda N, Komuro I. Genetic basis of hereditary thoracic aortic aneurysms and dissections. J Cardiol 2019;74(02):136-143

12 Guo DC, Regalado ES, Pinard A, et al; University of Washington Center for Mendelian Genomics. LTBP3 pathogenic variants predispose individuals to thoracic aortic aneurysms and dissections. Am J Hum Genet 2018;102(04):706-712

13 Morkmued S, Hemmerle J, Mathieu E, et al. Enamel and dental anomalies in latent-transforming growth factor beta-binding protein 3 mutant mice. Eur J Oral Sci 2017;125(01):8-17

14 Dabovic B, Chen Y, Colarossi C, et al. Bone abnormalities in latent TGF-[beta] binding protein (LTBP)-3-null mice indicate a role for Ltbp-3 in modulating TGF-[beta] bioavailability. J Cell Biol 2002; 156(02):227-232

15 Tan KL, Haelterman NA, Kwartler CS, et al; University of Washington Center for Mendelian Genomics. Ari-1 regulates myonuclear organization together with Parkin and is associated with aortic aneurysms. Dev Cell 2018;45(02):226-244.e8 
16 Corbitt H, Morris SA, Gravholt $\mathrm{CH}$, et al; GenTAC Registry Investigators. TIMP3 and TIMP1 are risk genes for bicuspid aortic valve and aortopathy in Turner syndrome. PLoS Genet 2018;14 (10):e1007692

17 Shalata A, Mahroom M, Milewicz DM, et al. Fatal thoracic aortic aneurysm and dissection in a large family with a novel MYLK gene mutation: delineation of the clinical phenotype. Orphanet J Rare Dis 2018;13(01):41

18 Nogi M, Satoh K, Sunamura S, et al. Small GTP-binding protein GDP dissociation stimulator prevents thoracic aortic aneurysm formation and rupture by phenotypic preservation of aortic smooth muscle cells. Circulation 2018;138(21):2413-2433

19 Kwartler CS, Gong L, Chen J, et al. Variants of unknown significance in genes associated with heritable thoracic aortic disease can be low penetrant "risk variants". Am J Hum Genet 2018;103 (01):138-143

20 Milewicz DM, Prakash SK, Ramirez F. Therapeutics targeting drivers of thoracic aortic aneurysms and acute aortic dissections: insights from predisposing genes and mouse models. Annu Rev Med 2017;68:51-67

21 Milewicz D, Hostetler E, Wallace S, et al. Precision medical and surgical management for thoracic aortic aneurysms and acute aortic dissections based on the causative mutant gene. J Cardiovasc Surg (Torino) 2016;57(02):172-177

22 Bradley TJ, Bowdin SC, Morel CF, Pyeritz RE. The expanding clinical spectrum of extracardiovascular and cardiovascular manifestations of heritable thoracic aortic aneurysm and dissection. Can J Cardiol 2016;32(01):86-99

23 Disabella E, Grasso M, Gambarin FI, et al. Risk of dissection in thoracic aneurysms associated with mutations of smooth muscle alpha-actin 2 (ACTA2). Heart 2011;97(04):321-326

24 Guo DC, Pannu H, Tran-Fadulu V, et al. Mutations in smooth muscle alpha-actin (ACTA2) lead to thoracic aortic aneurysms and dissections. Nat Genet 2007;39(12):1488-1493

25 Andelfinger G, Loeys B, Dietz H. A decade of discovery in the genetic understanding of thoracic aortic disease. Can J Cardiol 2016;32(01):13-25

26 Heegaard AM, Corsi A, Danielsen CC, et al. Biglycan deficiency causes spontaneous aortic dissection and rupture in mice. Circulation 2007;115(21):2731-2738

27 Meester JA, Vandeweyer G, Pintelon I, et al. Loss-of-function mutations in the X-linked biglycan gene cause a severe syndromic form of thoracic aortic aneurysms and dissections. Genet Med 2017;19(04):386-395

28 Schwarze U, Hata R, McKusick VA, et al. Rare autosomal recessive cardiac valvular form of Ehlers-Danlos syndrome results from mutations in the COL1A2 gene that activate the nonsensemediated RNA decay pathway. Am J Hum Genet 2004;74(05): 917-930

29 Smith LB, Hadoke PW, Dyer E, et al. Haploinsufficiency of the murine Col3a1 locus causes aortic dissection: a novel model of the vascular type of Ehlers-Danlos syndrome. Cardiovasc Res 2011;90(01):182-190

30 D'hondt S, Guillemyn B, Syx D, et al. Type III collagen affects dermal and vascular collagen fibrillogenesis and tissue integrity in a mutant Col3a1 transgenic mouse model. Matrix Biol 2018; 70:72-83

31 De Paepe A, Malfait F. The Ehlers-Danlos syndrome, a disorder with many faces. Clin Genet 2012;82(01):1-11

32 Germain DP. Ehlers-Danlos syndrome type IV. Orphanet J Rare Dis 2007;2:32

33 Erbel R, Aboyans V, Boileau C, et al; ESC Committee for Practice Guidelines; The Task Force for the Diagnosis and Treatment of Aortic Diseases of the European Society of Cardiology (ESC). 2014 ESC Guidelines on the diagnosis and treatment of aortic diseases: document covering acute and chronic aortic diseases of the thoracic and abdominal aorta of the adult. Eur Heart J 2014; 35(41):2873-2926
34 Monroe GR, Harakalova M, van der Crabben SN, et al. Familial Ehlers-Danlos syndrome with lethal arterial events caused by a mutation in COL5A1. Am J Med Genet A 2015;167(06): 1196-1203

35 Mehta S, Dhar SU, Birnbaum Y. Common iliac artery aneurysm and spontaneous dissection with contralateral iatrogenic common iliac artery dissection in classic Ehlers-Danlos syndrome. Int J Angiol 2012;21(03):167-170

36 Wenstrup RJ, Meyer RA, Lyle JS, et al. Prevalence of aortic root dilation in the Ehlers-Danlos syndrome. Genet Med 2002;4(03): 112-117

37 Park AC, Phan N, Massoudi D, et al. Deficits in Col5a2 expression result in novel skin and adipose abnormalities and predisposition to aortic aneurysms and dissections. Am J Pathol 2017;187 (10):2300-2311

38 Huang J, Davis EC, Chapman SL, et al. Fibulin-4 deficiency results in ascending aortic aneurysms: a potential link between abnormal smooth muscle cell phenotype and aneurysm progression. Circ Res 2010;106(03):583-592

39 Igoucheva O, Alexeev V, Halabi CM, et al. Fibulin-4 E57K knock-in mice recapitulate cutaneous, vascular and skeletal defects of recessive cutis laxa 1B with both elastic fiber and collagen fibril abnormalities. J Biol Chem 2015;290(35):21443-21459

40 Loeys B, De Paepe A, Urban Z. EFEMP2-related cutis laxa. In: Pagon RA, Adam MP, Ardinger HH, et al., eds. GeneReviews ${ }^{\circledR}$. Seattle, WA1993

41 Jelsig AM, Urban Z, Hucthagowder V, Nissen H, Ousager LB. Novel ELN mutation in a family with supravalvular aortic stenosis and intracranial aneurysm. Eur J Med Genet 2017;60(02):110-113

42 Callewaert B, Renard M, Hucthagowder V, et al. New insights into the pathogenesis of autosomal-dominant cutis laxa with report of five ELN mutations. Hum Mutat 2011;32(04):445-455

43 Szabo Z, Crepeau MW, Mitchell AL, et al. Aortic aneurysmal disease and cutis laxa caused by defects in the elastin gene.J Med Genet 2006;43(03):255-258

44 Capuano A, Bucciotti F, Farwell KD, et al. Diagnostic exome sequencing identifies a novel gene, EMILIN1, associated with autosomal-dominant hereditary connective tissue disease. Hum Mutat 2016;37(01):84-97

45 Pereira L, Andrikopoulos K, Tian J, et al. Targeting of the gene encoding fibrillin-1 recapitulates the vascular aspect of Marfan syndrome. Nat Genet 1997;17(02):218-222

46 Pereira L, Lee SY, Gayraud B, et al. Pathogenetic sequence for aneurysm revealed in mice underexpressing fibrillin-1. Proc Natl Acad Sci U S A 1999;96(07):3819-3823

47 Judge DP, Biery NJ, Keene DR, et al. Evidence for a critical contribution of haploinsufficiency in the complex pathogenesis of Marfan syndrome. J Clin Invest 2004;114(02):172-181

48 Habashi JP, Judge DP, Holm TM, et al. Losartan, an AT1 antagonist, prevents aortic aneurysm in a mouse model of Marfan syndrome. Science 2006;312(5770):117-121

49 Lima BL, Santos EJ, Fernandes GR, et al. A new mouse model for Marfan syndrome presents phenotypic variability associated with the genetic background and overall levels of Fbn1 expression. PLoS One 2010;5(11):e14136

50 Morris SA, Orbach DB, Geva T, Singh MN, Gauvreau K, Lacro RV. Increased vertebral artery tortuosity index is associated with adverse outcomes in children and young adults with connective tissue disorders. Circulation 2011;124(04):388-396

51 Hiratzka LF, Bakris GL, Beckman JA, et al; American College of Cardiology Foundation/American Heart Association Task Force on Practice Guidelines; American Association for Thoracic Surgery; American College of Radiology; American Stroke Association; Society of Cardiovascular Anesthesiologists; Society for Cardiovascular Angiography and Interventions; Society of Interventional Radiology; Society of Thoracic Surgeons; Society for Vascular Medicine. 2010 ACCF/AHA/AATS/ACR/ASA/SCA/SCAI/SIR/STS/ SVM Guidelines for the diagnosis and management of patients 
with thoracic aortic disease. A Report of the American College of Cardiology Foundation/American Heart Association Task Force on Practice Guidelines, American Association for Thoracic Surgery, American College of Radiology, American Stroke Association, Society of Cardiovascular Anesthesiologists, Society for Cardiovascular Angiography and Interventions, Society of Interventional Radiology, Society of Thoracic Surgeons, and Society for Vascular Medicine. J Am Coll Cardiol 2010;55(14):e27-e129

52 Takeda N, Morita H, Fujita D, et al. Congenital contractural arachnodactyly complicated with aortic dilatation and dissection: case report and review of literature. Am J Med Genet A 2015;167A(10):2382-2387

53 Retailleau K, Arhatte M, Demolombe S, et al. Smooth muscle filamin $\mathrm{A}$ is a major determinant of conduit artery structure and function at the adult stage. Pflugers Arch 2016;468(07): $1151-1160$

54 Feng Y, Chen MH, Moskowitz IP, et al. Filamin A (FLNA) is required for cell-cell contact in vascular development and cardiac morphogenesis. Proc Natl Acad Sci U S A 2006;103(52): 19836-19841

55 Reinstein E, Frentz S, Morgan T, et al. Vascular and connective tissue anomalies associated with X-linked periventricular heterotopia due to mutations in Filamin A. Eur J Hum Genet 2013;21 (05):494-502

56 Lange M, Kasper B, Bohring A, et al. 47 patients with FLNA associated periventricular nodular heterotopia. Orphanet J Rare Dis 2015;10:134

57 Kuang SQ, Medina-Martinez O, Guo DC, et al. FOXE3 mutations predispose to thoracic aortic aneurysms and dissections. J Clin Invest 2016;126(03):948-961

58 Vermeer AMC, Lodder EM, Thomas D, et al. Dilation of the aorta ascendens forms part of the clinical spectrum of HCN4 mutations. J Am Coll Cardiol 2016;67(19):2313-2315

59 Lee VS, Halabi CM, Hoffman EP, et al; Brigham Genomic Medicine. Loss of function mutation in LOX causes thoracic aortic aneurysm and dissection in humans. Proc Natl Acad Sci U S A 2016;113(31):8759-8764

60 Hornstra IK, Birge S, Starcher B, Bailey AJ, Mecham RP, Shapiro SD. Lysyl oxidase is required for vascular and diaphragmatic development in mice. J Biol Chem 2003;278(16):14387-14393

61 Mäki JM, Räsänen J, Tikkanen $\mathrm{H}$, et al. Inactivation of the lysyl oxidase gene Lox leads to aortic aneurysms, cardiovascular dysfunction, and perinatal death in mice. Circulation 2002;106 (19):2503-2509

62 Ren W, Liu Y, Wang X, et al. $\beta$-Aminopropionitrile monofumarate induces thoracic aortic dissection in C57BL/6 mice. Sci Rep 2016; 6:28149

63 Guo DC, Gong L, Regalado ES, et al; GenTAC Investigators, National Heart, Lung, and Blood Institute Go Exome Sequencing Project; Montalcino Aortic Consortium. MAT2A mutations predispose individuals to thoracic aortic aneurysms. Am J Hum Genet 2015;96(01):170-177

64 Combs MD, Knutsen RH, Broekelmann TJ, et al. Microfibrilassociated glycoprotein 2 (MAGP2) loss of function has pleiotropic effects in vivo. J Biol Chem 2013;288(40):28869-28880

65 Barbier M, Gross MS, Aubart M, et al. MFAP5 loss-of-function mutations underscore the involvement of matrix alteration in the pathogenesis of familial thoracic aortic aneurysms and dissections. Am J Hum Genet 2014;95(06):736-743

66 Bellini C, Wang S, Milewicz DM, Humphrey JD. Myh11(R247C/R247C) mutations increase thoracic aorta vulnerability to intramural damage despite a general biomechanical adaptivity. J Biomech 2015;48(01):113-121

67 Pannu H, Tran-Fadulu V, Papke CL, et al. MYH11 mutations result in a distinct vascular pathology driven by insulin-like growth factor 1 and angiotensin II. Hum Mol Genet 2007;16(20): 2453-2462
68 Wang L, Guo DC, Cao J, et al. Mutations in myosin light chain kinase cause familial aortic dissections. Am J Hum Genet 2010; 87(05):701-707

69 Hannuksela M, Stattin EL, Klar J, et al. A novel variant in MYLK causes thoracic aortic dissections: genotypic and phenotypic description. BMC Med Genet 2016;17(01):61

70 Luyckx I, Proost D, Hendriks JMH, et al. Two novel MYLK nonsense mutations causing thoracic aortic aneurysms/dissections in patients without apparent family history. Clin Genet 2017;92 (04):444-446

71 McKellar SH, Tester DJ, Yagubyan M, Majumdar R, Ackerman MJ, Sundt TM III. Novel NOTCH1 mutations in patients with bicuspid aortic valve disease and thoracic aortic aneurysms. J Thorac Cardiovasc Surg 2007;134(02):290-296

72 Proost D, Vandeweyer G, Meester JA, et al. Performant mutation identification using targeted next-generation sequencing of 14 thoracic aortic aneurysm genes. Hum Mutat 2015;36(08): 808-814

73 Guo DC, Regalado E, Casteel DE, et al; GenTAC Registry Consortium; National Heart, Lung, and Blood Institute Grand Opportunity Exome Sequencing Project. Recurrent gain-of-function mutation in PRKG1 causes thoracic aortic aneurysms and acute aortic dissections. Am J Hum Genet 2013;93(02):398-404

74 Doyle AJ, Doyle JJ, Bessling SL, et al. Mutations in the TGF- $\beta$ repressor SKI cause Shprintzen-Goldberg syndrome with aortic aneurysm. Nat Genet 2012;44(11):1249-1254

75 Callewaert BL, Willaert A, Kerstjens-Frederikse WS, et al. Arterial tortuosity syndrome: clinical and molecular findings in 12 newly identified families. Hum Mutat 2008;29(01):150-158

76 Micha D, Guo DC, Hilhorst-Hofstee Y, et al. SMAD2 mutations are associated with arterial aneurysms and dissections. Hum Mutat 2015;36(12):1145-1149

77 Zhang W, Zeng $\mathrm{Q}, \mathrm{Xu}$ Y, et al. Exome sequencing identified a novel SMAD2 mutation in a Chinese family with early onset aortic aneurysms. Clin Chim Acta 2017;468:211-214

78 Tan CK, Tan EH, Luo B, et al. SMAD3 deficiency promotes inflammatory aortic aneurysms in angiotensin II-infused mice via activation of iNOS. J Am Heart Assoc 2013;2(03):e000269

79 van der Linde D, van de Laar IM, Bertoli-Avella AM, et al. Aggressive cardiovascular phenotype of aneurysms-osteoarthritis syndrome caused by pathogenic SMAD3 variants. J Am Coll Cardiol 2012;60(05):397-403

80 van de Laar IM, van der Linde D, Oei EH, et al. Phenotypic spectrum of the SMAD3-related aneurysms-osteoarthritis syndrome. J Med Genet 2012;49(01):47-57

81 Zhang P, Hou S, Chen J, et al. Smad4 deficiency in smooth muscle cells initiates the formation of aortic aneurysm. Circ Res 2016; 118(03):388-399

82 Heald B, Rigelsky C, Moran R, et al. Prevalence of thoracic aortopathy in patients with juvenile polyposis syndrome-hereditary hemorrhagic telangiectasia due to SMAD4. Am J Med Genet A $2015 ; 167 \mathrm{~A}(08): 1758-1762$

83 Wain KE, Ellingson MS, McDonald J, et al. Appreciating the broad clinical features of SMAD4 mutation carriers: a multicenter chart review. Genet Med 2014;16(08):588-593

84 Gillis E, Kumar AA, Luyckx I, et al; Mibava Leducq Consortium. Candidate gene resequencing in a large bicuspid aortic valveassociated thoracic aortic aneurysm cohort: SMAD6 as an important contributor. Front Physiol 2017;8:400

85 Lindsay ME, Schepers D, Bolar NA, et al. Loss-of-function mutations in TGFB2 cause a syndromic presentation of thoracic aortic aneurysm. Nat Genet 2012;44(08):922-927

86 Boileau C, Guo DC, Hanna N, et al; National Heart, Lung, and Blood Institute (NHLBI) Go Exome Sequencing Project. TGFB2 mutations cause familial thoracic aortic aneurysms and dissections associated with mild systemic features of Marfan syndrome. Nat Genet 2012;44(08):916-921 
87 Renard M, Callewaert B, Malfait F, et al. Thoracic aortic-aneurysm and dissection in association with significant mitral valve disease caused by mutations in TGFB2. Int J Cardiol 2013;165(03):584-587

88 Bertoli-Avella AM, Gillis E, Morisaki H, et al. Mutations in a TGF- $\beta$ ligand, TGFB3, cause syndromic aortic aneurysms and dissections. J Am Coll Cardiol 2015;65(13):1324-1336

89 Gallo EM, Loch DC, Habashi JP, et al. Angiotensin II-dependent TGF- $\beta$ signaling contributes to Loeys-Dietz syndrome vascular pathogenesis. J Clin Invest 2014;124(01):448-460

90 MacCarrick G, Black JH III, Bowdin S, et al. Loeys-Dietz syndrome: a primer for diagnosis and management. Genet Med 2014;16 (08):576-587

91 Jondeau G, Ropers J, Regalado E, et al; Montalcino Aortic Consortium. International registry of patients carrying TGFBR1 or TGFBR2 mutations: results of the MAC (Montalcino Aortic Consortium). Circ Cardiovasc Genet 2016;9(06):548-558

92 Boodhwani M, Andelfinger G, Leipsic J, et al; Canadian Cardiovascular Society. Canadian Cardiovascular Society position statement on the management of thoracic aortic disease. Can J Cardiol 2014;30(06):577-589

93 Attias D, Stheneur C, Roy C, et al. Comparison of clinical presentations and outcomes between patients with TGFBR2 and FBN1 mutations in Marfan syndrome and related disorders. Circulation 2009;120(25):2541-2549

94 Teixidó-Tura G, Franken R, Galuppo V, et al. Heterogeneity of aortic disease severity in patients with Loeys-Dietz syndrome. Heart 2016;102(08):626-632

95 Tran-Fadulu V, Pannu H, Kim DH, et al. Analysis of multigenerational families with thoracic aortic aneurysms and dissections due to TGFBR1 or TGFBR2 mutations. J Med Genet 2009;46(09):607-613

96 Wenstrup RJ, Florer JB, Davidson JM, et al. Murine model of the Ehlers-Danlos syndrome. col5a1 haploinsufficiency disrupts collagen fibril assembly at multiple stages. J Biol Chem 2006; 281(18):12888-12895
97 Park AC, Phillips CL, Pfeiffer FM, et al. Homozygosity and heterozygosity for null Col5a2 alleles produce embryonic lethality and a novel classic Ehlers-Danlos syndrome-related phenotype. Am J Pathol 2015;185(07):2000-2011

98 Todorovic V, Frendewy D, Gutstein DE, Chen Y, Freyer L, Finnegan E, Rifkin DB. Long form of latent TGF-beta binding protein 1 Ltbp1L) is essential for cardiac outflow tract septation and remodeling. Development 2007;134:3723-3732

99 Kuang SQ, Kwartler CS, Byanova KL, et al. Rare, nonsynonymous variant in the smooth muscle-specific isoform of myosin heavy chain, MYH11, R247C, alters force generation in the aorta and phenotype of smooth muscle cells. Circ Res 2012;110(11): 1411-1422

100 Koenig SN, LaHaye S, Feller JD, et al. Notch1 haploinsufficiency causes ascending aortic aneurysms in mice. JCI Insight 2017;2 (21):91353

101 Berk M, Desai SY, Heyman HC, Colmenares C. Mice lacking the ski proto-oncogene have defects in neurulation, craniofacial, patterning, and skeletal muscle development. Genes Dev 1997;11 (16):2029-2039

102 Zoppi N, Chiarelli N, Cinquina V, Ritelli M, Colombi M. GLUT10 deficiency leads to oxidative stress and non-canonical $\alpha v \beta 3$ integrin-mediated TGF $\beta$ signalling associated with extracellular matrix disarray in arterial tortuosity syndrome skin fibroblasts. Hum Mol Genet 2015;24(23):6769-6787

103 Cheng $\mathrm{CH}$, Kikuchi T, Chen YH, et al. Mutations in the SLC2A10 gene cause arterial abnormalities in mice. Cardiovasc Res 2009; 81(02):381-388

104 Galvin KM, Donovan MJ, Lynch CA, et al. A role for smad6 in development and homeostasis of the cardiovascular system. Nat Genet 2000;24(02):171-174

105 Azhar M, Schultz Jel J, Grupp I, et al. Transforming growth factor beta in cardiovascular development and function. Cytokine Growth Factor Rev 2003;14(05):391-407 